\title{
Kernos
}

Revue internationale et pluridisciplinaire de religion grecque antique

7| 1994

Varia

\section{Hellenoaegyptiaca I : Influences égyptiennes dans l'imaginaire grec de la mort. Quelques exemples d'un emprunt supposé (Diodore, I, 92, 1-4 ; I, 96, 4-8)}

Francesco Diez de Velasco et Miguel Angel Molinero Polo

\section{OpenEdition}

Édition électronique

URL : http://journals.openedition.org/kernos/1097

DOI : 10.4000/kernos. 1097

ISSN : 2034-7871

Éditeur

Centre international d'étude de la religion grecque antique

Édition imprimée

Date de publication : 1 janvier 1994

ISSN : 0776-3824

Référence électronique

Francesco Diez de Velasco et Miguel Angel Molinero Polo, « Hellenoaegyptiaca I : Influences égyptiennes dans l'imaginaire grec de la mort. Quelques exemples d'un emprunt supposé (Diodore, I, 92, 1-4 ; I, 96, 4-8) », Kernos [En ligne], 7 | 1994, mis en ligne le 20 avril 2011, consulté le 20 avril 2019. URL : http://journals.openedition.org/kernos/1097 ; DOI : 10.4000/kernos.1097 
Kernos, 7 (1994), p. 75-93.

\title{
HELLENOAEGYPTIACA I
}

\section{INFLUENCES ÉGYPTIENNES \\ DANS L'IMAGINAIRE GREC DE LA MORT : QUELQUES EXEMPLES DUN EMPRUNT SUPPOSÉ}

(DIODORE, I, 92, 1-4; I, 96, 4-8)

\begin{abstract}
Diodore de Sicile, présente dans le livre I $(92,1-4 ; 96,4-8)$ de sa Bibliothèque Historique une vision des pratiques mortuaires égyptiennes qu'il définit comme antérieures et directement à l'origine des idées grecques du voyage vers l'au-delà. Une attitude naïve envers ces témoignages et ceux d'autres sources littéraires pourrait entraîner dans la voie sans issue d'un "pan-égyptisme" mal compris comme c'est le cas des récents travaux de Martin Bernal ${ }^{1}$. Dans les lignes qui suivent nous présentons une tentative pour déterminer si le témoignage de Diodore peut servir à défendre la thèse d'un emprunt réel à l'Égypte ou s'il ne s'agit que d'un emprunt supposé.
\end{abstract}

\section{Abréviations}

Burton = A. Burton, Diodorus Siculus. Book I. Commentary, Leiden, 1972.

CT = A. Buck, The Egyptian Coffin Texts, Chicago, 1935-1961, 7 vol.

Goyon = J.-C. Goyon, Rituels funéraires de l'ancienne Égypte, Paris, 1972.

$L \ddot{A}=$ Lexikon der Ägyptologie (W. HELCK, W. WESTENDORF, eds.), Wiesbaden, 7 vol. 1972 et suiv.

$R d E=$ Revue d'Égyptologie, Paris.

SEEBER = Ch. SEEBER, Untersuchungen zur Darstellung des Totengerichts im Alten Aegypten, München, 1976.

Vercoutter $=$ J. Vercoutter, Textes biographiques du Sérapéum de Memphis. Contribution à l'étude des stèles votives du Sérapéum, Paris, 1962.

Wb. = A. ERMAN, H. GRAPOW, Wörterbuch der ägyptischen Sprache, Berlin, 19261931,7 vol.

1 M. Bernal, Black Athena. The Afroasiatic Roots of Classical Civilization, I. The Fabrication of Ancient Greece 1785-1985, Londres, 1987; II. The Archaeological and Documentary Evidence, Londres, 1991. Il annonce un volume IV sur des questions mythologiques et religieuses. 


\section{Les eaux infernales et le nocher}

Il faut commencer par remarquer l'importance de l'eau dans les cultures grecque et égyptienne. Les eaux du Nil, raison d'être de l'Égypte, plus présentes encore lors de la crue qui transforme le pays en un monde d'îles, donnèrent lieu à une culture dont une évidente adaptation écologique conforma l'imaginaire cosmique et eschatologique. La vie des dieux et des défunts se déroule entre courants d'eau et marécages, tout comme dans le monde des vivants, autour d'un Nil de source cosmique et éternelle ${ }^{2}$. Les Grecs, pour leur part, donnèrent une importance à l'eau que Rudhardt ${ }^{3}$ a bien mis en évidence, eau primordiale qui entoure la terre, qui pénètre dans le monde inférieur et détermine l'existence des eaux infernales ${ }^{4}$ que le défunt, au moins à partir d'une certaine époque, est censé franchir5. Peuple de navigateurs, habitant un territoire formé de terres ouvertes à la mer et d'îles, l'influence du milieu semble suffisante pour comprendre l'importance que revêt l'eau dans l'imaginaire des temps primordiaux et de l'au-delà chez les Grecs.

Pourtant la narration de Diodore $(I, 96,7)$ se réfère à un monde réel 6 :

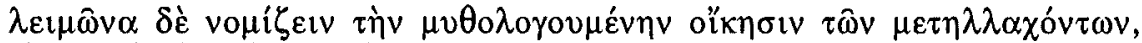

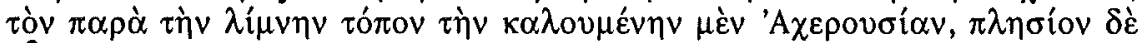

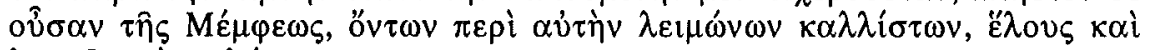

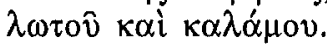

...il considère comme une prairie la mythique demeure des défunts : c'est le lieu situé au bord du lac nommé Achérousia, proche de Memphis auprès duquel se trouvent de magnifiques prairies et un marais, des lotus et des roseaux.

2 D. KURT, $L \ddot{A}$, IV, 485-489 s.v. "Nilgott»; S. SAUneron, J. YoYotTE, $L a$ naissance du monde selon l'Égypte ancienne, in La naissance du monde, Paris, 1959 (Sources Orientales, 1).

3 J. RUDHARDT, Le thème de l'eau primordiale dans la mythologie grecque, Berne, 1971, sur Okéanos cf. spécialement p. 50; voir aussi M. NINCK, Die Bedeutung des Wassers im Kultus und Leben der Alten, Leipzig, 1921, chap. 1.

4 HÉs., Th., 787 sq., entre autres.

5 Le passage dans l'au-dela n'est pas aquatique, par exemple, dans la deuteronekyia de l'Odyssée (XXIV, 1 sq.).

6 Nous suivons dans les citations la traduction de Y. Vernière et le texte grec établi par P. BERTRAC, Diodore de Sicile. Bibliothèque historique. Livre I, Paris, 1992 (Budé). 
Nous avons la certitude de l'existence à Memphis d'un canal artificiel qui reliait l'entrée du Fayoum au Delta et qui courait quelques kilomètres à l'Ouest, parallèle au Nil ${ }^{7}$. À hauteur de Memphis le canal, pendant l'inondation, devenait un lac qui s'étendait de la ville jusqu'à la falaise rocheuse au-dessus de laquelle se trouvait la nécropole. Les défunts qui étaient enterrés à Saqqarah venant de la ville devaient être transportés en barque à travers ce lac qui est de toute évidence celui que

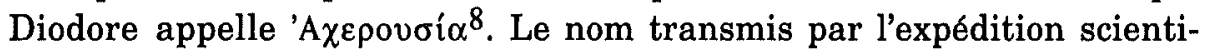
fique de Napoléon, au XIXe siècle, est Canal de el-Asara ${ }^{9}$, mais nous n'avons pas la certitude qu'il s'agisse de l'évolution phonétique du nom pharaonique malgré une certaine ressemblance avec celle de Diodore.

Le seul nom égyptien connu du lac est transmis par le Texte géographique d'Edfou ${ }^{10}$ et transcrit comme $\varphi \chi^{\prime} \tau^{11}$ dans les documents grecs. Il existait au temple de Mut à Karnak un lac appelé Acherou ${ }^{12}$, mais son rapport avec les faits racontés par Diodore semble difficile à établir. Ce premier argument de Diodore pour défendre les ressemblances entre les pratiques funéraires des deux peuples, le lac égyptien ('A $\chi \varepsilon \rho o v \sigma i ́ \alpha$ ) et la branche d'eau infernale des

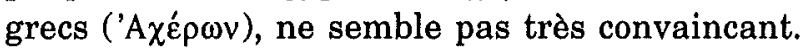

D'autre part l'identification que Diodore (I, 12, 5-6; I, 19, 4; I, 96, 7)

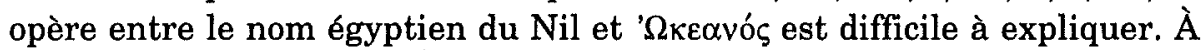
notre connaissance les Égyptiens n'avaient pas de nom précis pour le $\mathrm{Nil}$, qu'ils nommaient simplement «le grand fleuve», n3 itrw' 3 (Wb.,

7 G. Goyon, Les ports des pyramides et le grand canal de Memphis, in RdE, 23 (1971), p. 148-149. STRABON (XVII, 1, 32) en témoigne sans en donner le nom.

8 W. SPIEGELBERG, Ein Bruchstück des Bestattungsrituals des Apisstiere (Demot. Pap. Wien Nr. 27), in Zeitschrift für ägyptische Sprache und Altertumskunde, $56(1920)$, p. 19, note 7.

9 GoYoN (cité en note 7), p. 150.

10 H. GAUTHIER, Dictionnaire des noms géographiques contenus dans les textes hiéroglyphiques, 1925-1931 (reimp. Osnabrück 1975) IV, p. 188.

11 E. AMÉLINEAU, La géographie de l'Égypte à l'époque copte, 1893 (réimpr. Osnabrück, 1973), p. 340.

12 GoYoN, p. 171, 332. 
I, 146,17$)^{13}$. La source intarissable du Nil était Noun ${ }^{14}$, eau primordiale origine de toutes les eaux (souterraines, de pluie, les fleuves, les eaux qui entourent la terre, dans une multiplicité qui coexiste avec l'unité); l'analogie avec l'Océan primordial de la mythologie grecque (qui possède dans Homère des caractéristiques de Dieu-fleuve) a pu amener Diodore à forcer l'identité.

Le paysage que Diodore décrit dans les alentours du lac Acherousia peut correspondre à la géographie réelle, mais si nous relisons ses propres paroles (I, 96, 7), il est aisé de constater qu'il s'exprime d'une façon confuse :

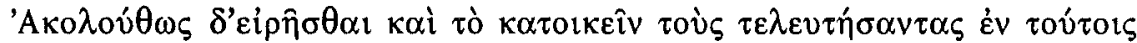

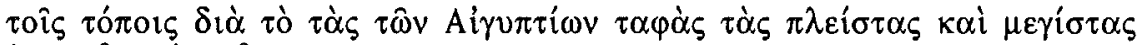

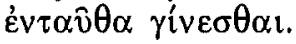

Cela s'accorde aussi avec la tradition qui place en ces lieux le séjour des morts car c'est là que se trouvent les plus nombreux et les plus grands tombeaux des Égyptiens.

Un mélange entre le monde imaginaire ("séjour des morts») et le monde réel («tombeaux des Égyptiens») semble clair. Toute cette description paraît plutôt nous introduire dans la Douat, l'au-delà constitué depuis l'Ancien Empire par un ensemble de prairies (Champ des Souchets, Champ des Offrandes, etc.) où le mort doit travailler en réalisant une éternelle et plaisante récolte.

Diodore dans deux localisations (I, 92, 2; I, 96, 8) rapporte que le nom $\mathrm{du}$ nocher grec Charon et ses fonctions sont un emprunt égyptien. Ce qui pour les Grecs est mythologie trouve, pour les Égyptiens, son explication dans la réalité quotidienne : accomplir le trajet entre la maison et la nécropole et qui nécessairement doit se faire en barque ${ }^{15}$. L'antiquité de

13 W. BUTZER, $L \ddot{A}$, IV, 480-483 s.v. «Nil»; en outre, la puissance fécondatrice du Nil était symbolisée par une divinité masculine à poitrine féminine dont le nom était Hâpy.

14 R. GRIEShammer, LÄ, IV, 534-535 s.v. «Nun»; SAUNERON-YOYOTTE (cité en note 2), p. 22 sq.

15 Certains ouvrages localisent les nécropoles égyptiennes dans le désert de l'Ouest et les villes de l'autre côté du Nil, ce qui n'est pas correct : on retrouve les tombeaux sur le plateau désertique dans les deux rivages tandis que les villes se situent près du fleuve (cf. D. KESSLER, $L \ddot{A}$, IV, 395-414 s.v. «Nekropolen. Frühzeit und AR»; F. GOMAA, LÄ, IV, 415-427 s.v. «Nekropolen des MR»; J. MÁLEK, LÄ, IV, 427-440 s.v. "Nekropolen. New Kingdom»; ID., LÄ, IV, 440-449 s.v. "Nekropolen. Late Period»). La cause véritable de l'emploi de 
la culture égyptienne et le fait que la pratique soit réelle et non imaginaire semblent suffisants à Diodore pour justifier le sens de l'emprunt.

Cependant Charon possède des caractéristiques tout à fait spécifiques qui semblent incompatibles avec une origine non grecque. Le poème épique Minyas en offre la première mention littéraire comme personnage mythologique. L'analyse des bribes qui ont survécu permet de le situer chronologiquement à la fin de la période archaïque et probablement dans un contexte géographique béotien ${ }^{16}$. L'iconographie apparaît vers 500 avec l'eschara de Frankfurt ${ }^{17}$ et le fragment de phormiskos de Tübingen ${ }^{18}$; la plus grande partie des représentations se développe sur des lécythes attiques à fond blanc et sur des exemplaires de qualité médiocre que devait acheter une clientèle populaire ${ }^{19}$. Personnage nonhomérique, il définit un imaginaire de la mort bien différent de l'imaginaire aristocratique qui revendique ses modèles à travers des héros épiques (l'adieu du guerrier - il faut lire l'hoplite -, l'exemple de Sarpédon). Charon, le nocher, vêtu comme un thes, dont le naulon est à la portée de tout le monde, devient le mirage de la mort facile des soutiens de la démocratie (les rameurs athéniens), de l'entrée de l'Hadès ouverte à tous les hommes, bien différent de l'Hadès homérique peuplé de membres des lignages héroïques.

Le système social qui préside à l'apparition de Charon (ou son remaniement, peut-être existait-il un génie populaire de la mort $a_{\text {térieur }}^{20}$ qui n'a pas pu nous parvenir car l'idéologie populaire n'a eu

bateaux dans les funérailles est que tout le paysage égyptien apparaissait traversé par des canalisations d'eau que les processions funéraires devaient franchir (pendant l'inondation la nécessité s'avérait plus évidente encore).

16 Minyas, fr. 1 (Bernabé); cf. F. DIEz DE VELASCO, Comentarios iconográficos y mitológicos del poema épico Miníada, in Gerion, 8 (1990), p. 73-87.

17 A. FURTWÄNGLER, Charon, eine altattische Malerei, in ARW, 8 (1905), p. 191202; K. DEPPERT, CVA Deutschland 30, Frankfurt 2, 1968, p. 11-12, ill. 46.

18 Interprété correctement par H. MOMMSEN, Irrfahrten des Odysseus. $Z u$ dem Fragment Tübingen s.10.1507, in Praestant Interna. Festschrift für Ulrich Haussmann, Tubingen, 1982, p. 209 et sq.

19 F. DIEZ DE VELASCO, El origen del mito de Caronte. Investigación sobre la idea popular del paso al más allá en la Atenas clásica, Madrid, 1988 (thèse doctorale, Université Complutense de Madrid).

20 Ce que défend H. HoffmanN, Charos, Charun, Charon, in OJA, 3 (1984), p. $65 \mathrm{sq} . ;$ ID., From Charos to Charon, some notes on the human encounter with death in attic red-figured vase-painting, in Visible Religion, 3 (1985), p. 173 sq.; dans une optique différente, F. DIEZ DE VELASCo, Aportación al 
des voies stables de pénétration dans la culture écrite ou figurée grecques qu'à partir de l'époque démocratique) est trop différent de celui qui conforme l'idéologie égyptienne de la mort, un système de redistribution centralisée et hautement bureaucratisée où même les biens funéraires sont un octroi de l'État.

Toutefois, une possibilité s'ouvre de justifier les paroles de Diodore. Dans l'imaginaire égyptien de la mort, plein de marécages et de courants d'eau, existe en effet un nocher qui embarque le mort. Sa dénomination dérive de la façon dont il apparaît : il regarde derrière lui pour voir le défunt qui embarque, mais tout en maintenant le corps dans le sens de la vogue. Il se nomme $H r . f-(m-) h 3 . f$, "Celui dont le visage est derrière lui» (Wb., III, 127, 11) ou M3-h3.f, "Celui qui regarde derrière lui" (Wb., II, 10,5 $)^{21}$, ce qui pourrait peut-être rappeler à un Grec la prononciation de Charon 22 . Le rapprochement de concepts

estudio del imaginario ático del paso al más allá : el genio psicopompo raptor del lécito Louvre CA 1264, in Héroes, semidioses y daimones, Coloquio ARYS 1989, Madrid, 1992, p. 59 sq.

21 On connaît trois groupes de dénominations du passeur : 1) mhnty, «celui de la barque» (Wb. , II, 133, 14-15) qui détermine son métier; 2 ) une série de noms développés à partir du verbe $m 33$ «voir» ou $h r$ «visage»; 3) une série d'autres noms, très variés, qui se rapportent à la fonction accomplie (Cf. Ch. JACQ, Le Voyage dans l'autre monde selon l'Égypte ancienne. Épreuves et métamorphoses du mort d'après les Textes des Pyramides et les Textes des Sarcophages, Monaco, 1986, 44-63). Les plus importants sont ceux qui sont tirés du deuxième groupe, notamment hr.f-h3.f et M3-h3.f. Il doit s'agir probablement d'un seul personnage dont le nom a été dédoublé depuis l'Ancien Empire. A cette époque la seule condition qu'il impose pour faire le trajet est de connaître le nom des pièces de la barque. A partir du Moyen Empire hr.f-h3.f acquiert une certaine connotation morale et reçoit l'épithète "celui qui aime la justice et déteste l'iniquité" (CT II, 138f-139a), ce qui peut expliquer son inclusion parmi les juges infernaux dans le chapitre 125 du Livre des Morts. D'après J. YOYOTTE, Le jugement des morts dans l'Égypte ancienne, in Le jugement des morts Paris, 1961 (Sources Orientales, 4), p. 64-65, ce texte aurait été établi pendant la période héracléopolitaine, il serait donc contemporain de la rédaction des Textes des Sarcophages. Dès le Nouvel Empire semble se préciser leur spécialisation, $h r . f-h 3 . f$ dans le rôle de juge et $M 3-h 3 . f$ dans celui de nocher, situation qui se maintiendra au moins jusqu'à l'époque grecque.

22 Rapprochement plus facile que celui que défend E. VERMEULE, Aspects of Death in Early Greek Art and Poetry, Los Angeles, 1979, p. 71, lorsqu'elle propose l'emprunt du modèle iconographique même du nocher égyptien car elle retrouve un Charon grec qui semble regarder derrière lui. Le document qu'elle présente est un fragment de lécythe attique à fond blanc de Munich (8925) qui est véritablement un hapax. Il fut publié par R. LuLLIES, "Charon", 
différents par le moyen de jouer d'une façon quelquefois forcée avec les homophonies se retrouve en Égypte, surtout à l'époque tardive, dans d'autres cas (l'écriture hiéroglyphique semble encourager ce genre d'exercices lorsqu'elle devient moins pratiquée à cause de l'emploi d'autres systèmes d'écriture - démotique, grec) ${ }^{23}$; l'œuvre de Diodore en est témoin dans plusieurs passages. Dans le chapitre douze du livre premier, il développe une théologie qui tente de rapprocher les divinités égyptiennes des dieux grecs sur la base de l'interprétation allégorique de leur relation avec les éléments constitutifs de la nature ${ }^{24}$.

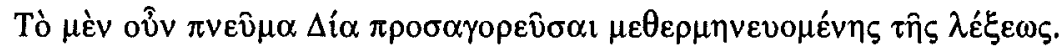

Ils appelèrent le souffle vital d'un nom qui, traduit dans notre langue, donne Zeus (I, 12, 2)

se comprend si au lieu de maintenir l'interpretatio graeca traditionnelle Zeus-Amon à l'origine de laquelle se trouve Hérodote ${ }^{25}$ nous

in $J D A I, 59-60$ (1944-45), p. 25 sq., ill. 19, 1, comme appartenant au peintre de Villa Giulia, ce que Beazley n'a pas confirmé. La scène est trop fragmentaire pour tirer des conclusions (seules la partie supérieure du corps du nocher et la rame subsistent), nous ne savons pas où se trouvait le défunt, mais effectivement le passeur regarde derrière lui. Le reste des documents connus (presque la centaine) présente une scène dans laquelle Charon regarde droit devant lui, normalement en direction du défunt (cf. DIEZ DE VELASCO [cité en note 19], p. 554 sq.); l'hypothèse de Vermeule semble vraiment peu fondée.

23 Par exemple P. DERCHAIN, Le démiurge et la balance, in Religions en Égypte hellénistique et romaine, Colloque de Strasbourg 1967, Paris 1969, p. 31 sq. BURTON, p. 65, parle du phénomène des allitérations qui est d'une nature un peu différente, mais son commentaire est envisagé dans une perspective différente de la nôtre et n'étudie pas les exemples d'homophonies que nous présentons. Cf. aussi les constants jeux de mots dans l'article de M. Broze dans ce même colloque.

24 Le passage complet a été étudié par W. SPOERRI, Späthellenistische Berichte über Welt, Kultur und Götter. Untersuchungen zur Diodor von Sizilien, Bâle, 1959 , p. 180 sq.

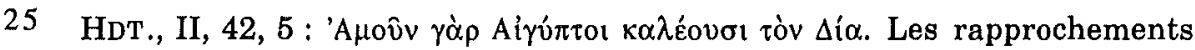
divins réalisés par Hérodote entre dieux égyptiens et grecs furent repris genéralement par Diodore. La bibliographie est imposante; cf. entre autres J. YoYOTTE, L'identification des dieux égyptiens à des dieux grecs : données et problèmes, in Formation et survie des mythes, Coll. Nanterre 1974, Paris, 1977, p. 37-39; J.M. LINFORTH, Greek and Egyptian Gods (Hdt. II, 50-52), in CPh, 35 (1940), p. 91-110; K.S. KoLTE, Die Gleichsetzung ägyptischer und griechischer Götter bei Herodot, Tubingen, 1968; W. B URKERT, Herodot über die Namen der Götter, Polytheismus als historisches Problem, in $M H, 42$ (1985), p. 121-132 ou le travail de G. Zographou dans ces actes. Plus généraux 
prononçons le nom égyptien de l'air et le souffle vital, $t 3 w$ (Wb., V, 350, 12), pas trop loin de Zeus du point de vue de la sonorité, encore moins à l'époque de Diodore puisque dans les textes coptes, le nom est devenu $\Theta$ HOY $^{26}$. Pour l'élément Terre, Diodore fait le rapprochement avec Déméter, tout en présentant une étymologie qu'il défend par l'inclusion d'une citation directe :

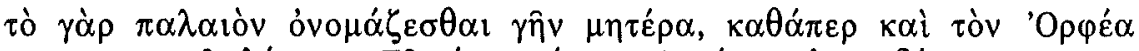

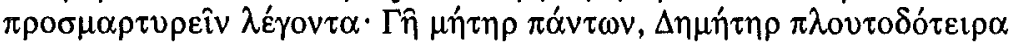

autrefois on l'appelait Gê Meter comme en témoigne Orphée lorsqu'il dit : (fr. 302 Kern) «Terre, mère de toutes choses, féconde Déméter».

D'après Diodore, les Égyptiens nomment la terre «mère» et c'est cette dénomination que les Grecs ont adoptée. L'homophonie de $G b$ (Geb, le dieu terre égyptien [Wb., V, 164, 6]) et Gê semble la base de la spéculation de Diodore, mais dans ce cas, le résultat n'est pas satisfaisant car le Geb égyptien est une divinité masculine qui peut donc être difficilement invoqué avec la qualité de mère. Le rapprochement par homophonie est moins évident dans le cas de l'élément air $(\mathrm{I}, 12,7)$ car il se produit un changement de place des syllabes des théonymes (Neith27-Athéna) même si la structure consonantique est identique, mais Diodore dans ce cas est cautionné par son prédécesseur Hérodote (II, 28).

En outre un emprunt sûr de la langue grecque à l'égyptienne, le mot baris $^{28}$, a pu donner encore plus d'arguments à Diodore pour risquer l'identification des deux nochers infernaux, bien que l'utilisation en grec de baris pour nommer la barque de Charon soit très $\operatorname{tardive}^{29}$ et que

C. Sourdille, Hérodote et la religion de l'Égypte, Paris, 1910; F. MorA, Religione e religioni nelle storie di Erodoto, Milan, 1985, ou W. BURKERT, Herodot als Historiker fremder Religionen, in Hérodote et les peuples non grecs, Genève-Vandœuvres, Fondation Hardt, 1990 (Entretiens de l'Antiquité classique, 35), p. 1-33, ou encore les excellents commentaires de A.B. LLOYD, Herodotus Book II, Introduction, Leyde, 1975; Commentary 1.98, Leyde, 1976; Commentary 99-182, Leyde, 1988, ou A.B. LLOYD, Herodotus on Egyptians and Libyans, in Hérodote et les peuples non grecs, GenèveVandœuvres, Fondation Hardt, 1990 (Entretiens de l'Antiquité classique, 35), p. 215-145.

26 Aussi THY dans d'autres manuscrits, $W b$, V, 350.

$27 N t$ en égyptien (Wb., II, 189, 9), Niï en grec.

28 P. Chantraine, Dictionnaire étymologique de la langue grecque, Paris, 1968, p. 165 s.v., cité aussi par HDT., II, 96.

29 Diccionario Griego-Español (DGE) (F.R. ADRADOS ed.) III, 1991 s.v. «baris». 
l'équivalent égyptien $b r(W b ., I, 465,8)$ serve pour se référer à toute sorte de bateau ${ }^{30}$ et non pas spécifiquement à la barque infernale.

En résumé, l'apparition du nocher s'explique en Égypte et en Grèce par leurs caractéristiques écologiques et socio-culturelles, sans le recours à l'emprunt ${ }^{31}$.

\section{Le jugement des morts}

On a déjà vu le rapport entre la description que fait Diodore des funérailles et la ville de Memphis. L'événement le plus important dans la vie de la nécropole memphite était la mise au tombeau de l'Apis.

Diodore semble bien informé en ce qui concerne les enterrements de ces taureaux. Au livre I, $84,7-8^{32}$, il parle du rôle important que jouaient certains prêtres, au moins dans l'organisation matérielle des funérailles, qu'ils finançaient même de leurs propres revenus. Or, quelques-unes des stèles découvertes par Mariette dans les souterrains du Sérapéum ont confirmé la part prise par certains personnages dans les rites qui se déroulaient après la mort du taureau divin ${ }^{33}$.

$30 \mathrm{~Wb}$, , I, 465 indique que le terme définit un bateau aux multiples usages (voyage, transport de denrées, etc.).

31 Des passeurs de l'au-delà existent dans des sociétés tellement différentes et éloignées que les emprunts culturels semblent plus qu'improbables, cf. L.V. GRINSELL, The Ferrymann and his Fee: a Study in Ethnology, Archaeology and Tradition, in Folklore, 68 (1957), p. 257-269.

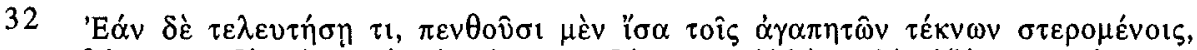

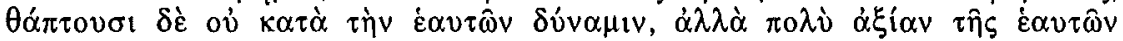

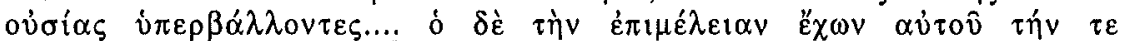

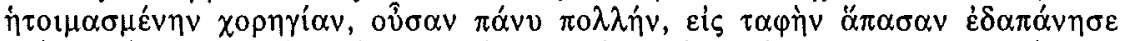

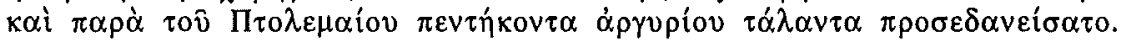

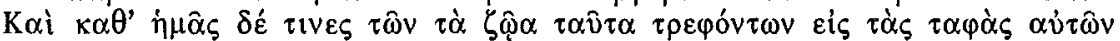

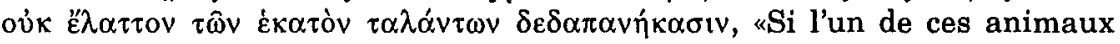
meurt, ils le pleurent comme s'ils avaient perdu un enfant chéri et ils l'ensevelissent non pas en fonction de leurs moyens, mais en allant bien audelà de leur situation de fortune... Celui qui avait soin de lui (le prêtre chargé de l'Apis) non seulement dépensa pour ses funérailles toute la somme, qui était tout à fait considérable, réservée à cet effet, mais encore emprunta à Ptolémée cinquante talents d'argent. Et même de nos jours, certains de ceux qui élèvent ces animaux n'ont pas dépensé moins de cent talents pour leurs funérailles".

33 Une stèle de l'an 4/5 du règne d'Amasis (SIM 4115 Louvre) nous rappelle : "Or, après le travail [d'embaumement] vint le jour de la privation [jeûne] (lorsque) le cadavre du dieu fut uni à la terre [enseveli], je lui donnai ses amulettes et ses ornements [qui étaient] en or et en toutes sortes de pierres 
Ces textes confirment qu'au moins sur ce point, on peut faire confiance à Diodore et imaginer qu'il devait avoir en tête les cérémonies de l'enterrement de l'Apis quand il rédigea tout ce passage ${ }^{34}$. Lorsque Diodore a fait son voyage en Égypte (entre les années 60-57 av. J.-C. ${ }^{35}$ ) le culte à l'Apis et ses funérailles avaient encore lieu ${ }^{36}$, et donc,

précieuses». (VERCOUTTER, p. 18 et 19, note $J$ et p. 20-26 pour la chronologie). Le texte garde sa valeur même si les mots sont pris textuellement des épitaphes officielles. Dans une autre stèle du règne de Darius I (SIM 4017 Louvre), le dédiant s'exprime ainsi : "Je suis un serviteur qui a agi pour ton $\mathrm{Ka}$, j'ai passé mes nuits à veiller chaque jour, je n'ai pas sommeillé, cherchant toutes [sortes de] choses utiles pour toi; j'ai mis la crainte que l'on a de toi dans le coeur de tout le monde [Égyptiens], et [dans celui] des étrangers de tous les pays étrangers qui étaient en Égypte étant donné ce que j'ai fait dans ta Ouabet. J'ai fait que partent [envoyés] les émissaires vers la Haute Égypte et de même ceux pour la Basse Égypte, pour faire venir tous les maires [et princes] des villes et des nomes avec leurs cadeaux pour ta Ouabet. Aussi les Pères divins et les prophètes du temple de Ptah furent-ils à dire : "Ô ApisOsiris, exauce la prière de celui qui a accompli tes rites, le Chef des Soldats Amasis; il a fait le bouclier [monté la garde] autour de toi, il est venu en personne apportant de l'argent, de l'or et du tissu royal, de la résine odoriférante, toutes espèces de pierres précieuses et toute sorte de bonnes choses, fais pour lui une récompense en proportion de ce qu'il a fait pour toi" (VERCOUTTER, p. 61). Amasis met en valeur le rôle personnel qu'il a joué dans la cérémonie : chef de la garde militaire qui accompagnait la procession funéraire et collecteur des dons indispensables à la momification, fournis à la demande de Memphis, par les nomes de toute l'Égypte.

34 BURTON, p. 270, avait déjà exprimé cette opinion, mais elle ne s'appuie pas sur ces stèles, même si elle mentionne le livre de Vercoutter dans sa bibliographie.

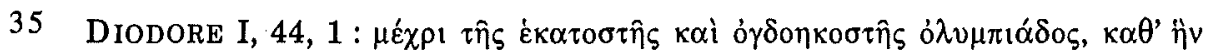

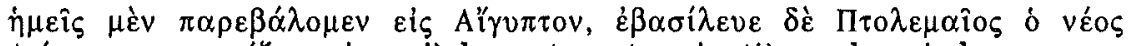

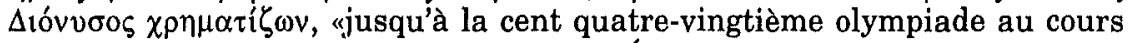
de laquelle nous nous sommes rendus en Égypte, sous le règne de Ptolémée qui prit le nom de nouveau Dionysos".

36 Les Grands Souterrains du Sérapéum ont été employés jusqu'à la fin de l'époque grecque (M. MAlinine, G. POSENER, J. VerCoutTEr, Catalogue des stèles du Sérapéum de Memphis, Paris, 1968, I, XII) mais quelques stèles témoignent de la survivance du culte encore plus longtemps. La dernière vache Isis, mère d'Apis, enterrée à l'Iséum date de l'année 11 de Cléopâtre VII (41 av. J.-C.; H. SMITH, Les catacombes des animaux sacrés, in Saqqara. Aux origines de l'Egypte pharaonique, in Les Dossiers d'Archéologie, 146-147 [1990], p. 117). L'information des sources littéraires s'étend davantage puisque DION CASSIUS $(51,16,5)$ et SUÉTONE (Aug., 93) rappellent le mépris d'Auguste envers le culte d'Apis; il refusa la visite lors de sa campagne victorieuse en Égypte, en affirmant qu'il adorait des dieux et non pas du bétail. Cf. M.A. Molinero Polo, C. Sevilla Cueva, Las dependencias cultuales del 
il a pu assister ou être renseigné de première main. L'enterrement de l'Apis suivait en tous les points le même rituel que celui d'un humain, mais avec beaucoup plus de faste et de richesse; il ne faut donc pas penser que Diodore a commis l'erreur de confondre les funérailles d'un homme avec celles d'un animal : elles étaient identiques.

Pour mieux comprendre la suite de Diodore, il faut s'attarder à expliquer quel était l'itinéraire que suivaient les dépouilles de l'Apis depuis sa mort jusqu'à la mise au tombeau. Vercoutter défend un parcours avec deux processions en se référant à des stèles biographiques découvertes par Mariette aux Souterrains : la première, après le décès de l'animal, allait du temple de Ptah, où se trouvait l'étable, jusqu'à la «Tente de purification», et de là à la Ouabet ${ }^{37}$, où le corps subissait tout le processus de la momification; la seconde se déroulait après le séjour de soixante-dix journées à la Ouabet lorsque la momie divine était conduite au "lac du Roi» où, semble-t-il, elle subissait une nouvelle purification dans un petit sanctuaire dressé au bord même de l'eau ${ }^{38}$. Puis, après avoir traversé en barque une certaine étendue d'eau (peutêtre le lac dont nous avons déjà parlé et que Diodore nommait Acherousia) ou un canal, la momie du dieu était placée sur un traîneau

buey sagrado de Menfis : revisión de una investigación arqueológica, in Espacio, Tiempo, Forma (ser. II), 6 (1993), sous presse.

37 «La Pure», la salle d'embaumement, a été identifiée il y a longtemps dans un sanctuaire à l'intérieur du grand temple de Ptah, juxtaposée à l'étable divine. Sur les restes de la Ouabet, cf. en dernier lieu M. JONES, The Temple of Apis in Memphis, in Journal of Egyptian Archaeology, 76 (1990), p. 141-147.

38 La stèle de l'an 23 d'Amasis (SIM 4110 Louvre) raconte : "Aucune nourriture n'est descendue dans mon estomac, si ce n'est du pain, de l'eau et des légumes, jusqu'à l'accomplissement des soixante-dix jours, jusqu'à ce que le grand dieu quitte la Ouabet. Puis Sa Majesté (Apis) sortit vers la Tente de Purification (?); lorsqu'elle descendit dans sa barque j'étais devant elle (ou à son avant, à sa proue) à gémir avec les "pauvres" (VERCOUTTER, p. 50). Neferibre était un courtisan de haut rang : Ami (Unique), Gouverneur du Palais, Confident de son Maître, Chef des Secrets Royaux. Vercoutter avance cette traduction avec quelques remarques à propos de la Tente de Purification (VERCOUTTER, p. 53, note $R$ ). Le signe employé (E 31 ) désigne habituellement la "salle d'embaumement", mais ce processus venait d'être accompli. Il pourrait s'agir

du mot $\int 2$ ibw (Wb., I, 62, 5), qui emploie aussi un signe qui représente une chèvre, bien qu'en tant que déterminatif et non comme phonogramme. Au pDem Wien 27 ce mot, écrit avec des signes unilitères, désigne une construction élevée aux bords du "Lac du Roi». Les restes de l'Apis, comme ceux des simples mortels, séjournaient deux fois dans la Tente. 
que l'on transportait jusqu'au Sérapéum. Ce n'est qu'à la proximité immédiate des souterrains ou dans son intérieur même qu'avait lieu la cérémonie de l'Ouverture de la Bouche, qui était censée transmettre aux défunts les facultés d'accès à la vie éternelle : ses organes retrouvaient leur fonctionnement et la vie réintégrait le cadavre ${ }^{39}$.

Les doutes de Vercoutter quant à la traduction du mot pour désigner soit la «Tente de Purification» traditionnelle soit une dépendance de la Ouabet désignée par les mêmes signes ne nuisent pas à notre interprétation. En tout cas, le mot désigne un élément architectural où l'on réalisait quelque rituel sur le corps du défunt. Reprenant la narration de Diodore, cette dépendance serait, selon toute toute vraisemblance celle que l'auteur grec appelle l'hémicycle et dans laquelle il situe les quarante-deux juges. Sur ce point, Diodore a dû mal interpréter le rituel qui s'y déroulait et auquel personne, excepté les prêtres et le personnel secondaire qui subissait une série de purifications, n'a pu assister. On a dû lui raconter qu'à l'intérieur se développait entre autres cerémonies, une lecture de textes religieux ${ }^{40}$ (avec, peut-être, les déclarations d'innocence) qu'il a dû confondre avec un jugement réel. Il aurait même pu s'agir d'une théâtralisation rituelle des épisodes de l'accès de l'âme à l'au-delà, car nous avons certains arguments pour défendre qu'une telle sorte de drame avait lieu en quelques cas. Une lettre du Sérapéum parle de deux filles qui sont maintenues aux frais du temple et sont utilisées pour représenter les pleureuses divines Isis et Nephthys dans les cérémonies des funérailles de l'Apis ${ }^{41}$. Yoyotte, de son côté, prétend que l'épisode d'Oknos dont parle Diodore (I, 97, 3) doit être l'interprétation d'une cérémonie mimée ${ }^{42}$. La façon de s'exprimer de Diodore dans I, 96, 6 nous permet encore plus clairement de défendre l'idée qu'il a pu connaître une réprésentation dramatique du voyage de

39 VERCOUTter, stèle $M$ et quelques autres exemples, p. 85, note H. Ils confirment toujours la similitude avec les rites accomplis sur les momies humaines.

40 Dans le papyrus de l'enterrement des Apis, on note la lecture de neuf papyrus au moment du transport du corps de l'animal dans la barque (SPIEGELBERG [cité en note 8], p. 19-22). Ce genre de lecture devait se dérouler aussi dans d'autres endroits et spécifiquement dans la Tente de Purification.

41 U. WILCKEN, Urkunden der Ptolemäerzeit (ältere Funde) I. Papyri aus Unterägypten. Berlin-Leipzig, 1927, p. 18 sq.

42 J. Yoyотте, Études géographiques. I. La Cité des Acacias (Kafr Ammar), in $R d E, 13$ (1961), p. 104-105. 
l'âme, mais la simultanéité subtile entre les actions en cours dans les deux mondes (réel-rituel et imaginaire) a dû lui échapper :

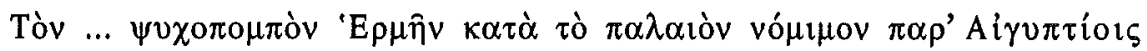

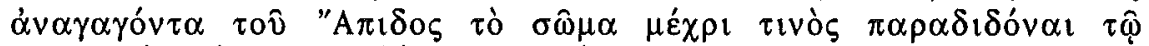

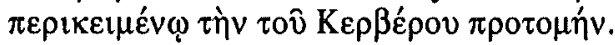

Hermès Psychopompe ..., selon l'antique coutume égyptienne, amène le corps d'Apis jusqu'à un certain lieu où il le remet à un personnage qui porte le masque de Cerbère [Anubis, dieu à la tête de chacal qu'on peut confondre avec un chien].

Il s'agit de la réception du cadavre du défunt par un prêtre nommé "Anubis supérieur des mystères" dans le Rituel de l'Embaumement (paragraphe VII), qui était le maître de cérémonies ${ }^{43}$ et dont nous supposons qu'il portait un masque de chacal du type de ceux qui sont parvenus jusqu'à nos jours ${ }^{44}$; Diodore a même pu les voir. Ce qui nous étonne dans sa narration, c'est que, dans la première partie de la phrase, il n'utilise pas le même procédé d'expression que dans la seconde; il aurait pu parler d'un personnage portant le masque d'Ibis et donc jouant le rôle de Thot (Hermès Psychopompe), mais, au contraire, il semble se référer à l'apparition propre du dieu. À ce point, il devient encore plus difficile de se faire une idée de ce que Diodore a pu voir lors de son voyage en Égypte et qui a contribué à la création de ce panorama composite qu'est sa narration des coutumes funéraires égyptiennes. Il aurait pu avoir vu un exemplaire du Livre des Morts avec la scène des quarante-deux juges présidant la confession négative (chap. 125), mais cela impliquerait qu'il aurait dû de même avoir vu la scène de la pesée du cœur (chap. 30) qui, dès la XIXe Dynastie, formait une unité avec la précédente ${ }^{45}$. À l'époque de Diodore, l'iconographie avait subi une évolution : les juges se trouvent déplacés dans la partie supérieure de la

43 Goyon, p. 26. Représentation d'un prêtre au masque de chacal dans A. MARIETTE, Denderah IV, Paris, 1873, ill, 31.

44 Par exemple Hildesheim, Pelizaeus Museum, 1585 (W. SEIPEL, Ägypten, Götter, Gräber und die Kunst. 4000 Jahre Jenseitsglaube, Linz, 1989, p. 158160). Cf. l'article monographique de A. WolinskI, Ancient Egyptian Ceremonial Masks, in Discussions in Egyptology, 6 (1986), p. 47-53.

45 Dans les Livres des Respirations (dont les exemplaires connus sont des siècles I-II, bien qu'un récit démotique romanesque fasse remonter la compilation à la dynastie saïte), la déclaration d'innocence se maintient comme texte indépendant mais le nombre des juges est descendu à 19 dans le manuscrit le plus complet (Londres, BM 9995). Diodore ne peut donc pas se référer à ces documents. GOYON, p. 191-196 et 224-226. 
salle et assistent à la scène principale qui est maintenant la pesée du cœur ${ }^{46}$, dont la représentation habituelle inclut la présence d'Anubis et de Thot (qui, dans certains cas, est investi du rôle de guide du mort jusqu'aux pieds de la balance ${ }^{47}$ ). Il semble évident que Diodore n'aurait pas résisté à faire le rapprochement entre la pesée du cœur et la psychostasie grecque ${ }^{48}$ s'il avait eu sous les yeux la scène, même s'il faut être conscient que, de toute façon, le seul aveu de la connaissance du Livre des Morts rendrait insoutenable une des thèses principales de l'auteur, à savoir que les Égyptiens développent des rituels funéraires réels là où les Grecs, en tant qu'adaptateurs, les estiment imaginaires.

En résumé, il semble plus utile de penser que Diodore, en ce qui concerne le jugement de la vie du mort et la présence de Cerbère et Hermès, a confondu une dramatisation rituelle faite par les prêtres lors des funérailles et qui rappelait ce qui était censé se produire dans le monde imaginaire, avec une cérémonie réelle.

L'emprunt grec des figures de Thot comme Hermès et d'Anubis comme Cerbère semble aussi peu probable que celui de Charon. À l'Hermès grec, en tant que dieu des limites ${ }^{49}$, correspond naturellement la seigneurie du passage entre le monde des vivants et celui des morts; la structure des fonctions du dieu dans l'ensemble du panthéon grec est si bien équilibrée que l'adjonction d'une mission étrange, moyennant un emprunt égyptien, aurait été perturbatrice. Le Cerbère grec, de son coté, est bien différent d'Anubis dans ses fonctions. Le dieu égyptien aide le mort dans le voyage vers l'au-delà et représente, bien plus que Thot, le psychopompe habituel ${ }^{50}$; le chien d'Hadès accomplit plutôt le travail contraire, c'est-à-dire qu'il symbolise les barrières de l'au-delà

\section{SEEBER, type E.}

47 Dans les sarcophages anthropoïdes de la Basse Époque (SEEBER, type D).

48 La psychostasie ou kérostasie grecque est de toute façon fonctionnellement différente puisqu'elle détermine dans un affrontement guerrier lequel des deux combattants est destiné à la victoire (Iliade, XXII, 208 sq.). L'iconographie des épisodes est très suggestive puisqu'elle se fonde sur des cuvres maintenant perdues, comme, par exemple, la Psychostasia d'Eschyle ( TrGF III F, 279-280a), cf. R. VOLLKOMMER, «Ker», in LIMC, VI (1992), 14 sq. ou E. PEIFER, Eidola, Frankfurt, 1989, p. 48 sq.

49 L. KAHN, Hermès passe ou les ambiguïtés de la communication, Paris, 1978.

50 Mais il n'est pas le seul; il y a, entre autres, Thot, Chou et Tefnout (dans le chap. 99 du Livre des Morts), Horus (SEEBER, type B1) et une déesse Maat ou une autre divinité (qui embrasse le défunt par le dos) dans les représentations tardives (SEEBER, type E). 
et le caractère monstrueux et radicalement "autre» du royaume infernal en tant qu'animal représentant le contraire de la civilisation $^{51}$.

\begin{tabular}{|l|l|l|l|}
\cline { 2 - 4 } \multicolumn{1}{c|}{} & \multicolumn{1}{c|}{$\begin{array}{c}\text { LÉgypte de } \\
\text { Diodore }\end{array}$} & \multicolumn{1}{c|}{ Grèce } & \multicolumn{1}{c|}{$\begin{array}{c}\text { Égypte } \\
\text { (autres sources) }\end{array}$} \\
\hline Jugement & $\begin{array}{l}\text { monde réel } \\
42 \text { juges }\end{array}$ & $\begin{array}{l}\text { monde imaginaire } \\
\text { Minos, Éaque... }\end{array}$ & $\begin{array}{l}\text { monde imaginaire } \\
42 \text { juges }\end{array}$ \\
\hline Lac & $\begin{array}{l}\text { monde réel } \\
\text { Achérousia }\end{array}$ & $\begin{array}{l}\text { monde imaginaire } \\
\text { Achéron }\end{array}$ & \\
\hline Nocher & $\begin{array}{l}\text { monde réel } \\
\text { Charon }\end{array}$ & $\begin{array}{l}\text { monde imaginaire } \\
\text { Charon }\end{array}$ & $\begin{array}{l}\text { monde imaginaire } \\
\text { Hr.f- }(m-) H 3 . f\end{array}$ \\
\hline Barque & $\begin{array}{l}\text { monde réel } \\
\text { baris }\end{array}$ & $\begin{array}{l}\text { baris (emprunt } \\
\text { égyptien) }\end{array}$ & $b r$ \\
\hline $\begin{array}{l}\text { Hermès } \\
\text { Psychopompe } \\
\text { Cerbère }\end{array}$ & $\begin{array}{l}\text { monde réel ? } \\
\text { Hermès } \\
\text { masque } \\
\text { de Cerbère }\end{array}$ & $\begin{array}{l}\text { monde imaginaire } \\
\text { Hermès, Cerbère }\end{array}$ & $\begin{array}{l}\text { monde imaginaire } \\
\text { Thot } \\
\text { Anubis }\end{array}$ \\
\hline
\end{tabular}

\section{Récapitulation - Conclusions}

De l'analyse de ce tableau, une conclusion semble s'imposer: Diodore, en ce qui concerne les rituels funéraires, défend l'idée selon laquelle ce qui chez les Égyptiens est réel, est devenu imaginaire chez les Grecs ${ }^{52}$, dégénérescence de coutumes qui, en Égypte, ont une véritable raison d'être. Il énumère les pratiques égyptiennes, les regardant avec les a priori de son époque et de sa formation, et les interprétant d'une façon qui révèle trois grandes influences.

La première provient de sa propre expérience comme visiteur grec d'un pays dont la culture n'est pas grecque. Coupé des sources directes, il a dû assister a des rituels qu'il ne comprenait que partiellement et qui lui étaient expliqués par des hellénisés qui, à leur tour, les inter-

51 C'est le chien semblable au loup, symbole des marginaux (les jeunes en initiation, les habitants des marges du territoire de la polis ...). Cf. C. MAINOLDI, Cani mitici e rituali tra il regno dei morti e il mondo dei viventi, in QUCC, 8 (1981), p. 7 sq.

52 D IODORE (I, 2, 2 et I, 93, 3) montre qu'il est conscient du caractère imaginaire de la mythologie infernale grecque. 
prétaient avec des outils au moins en partie étrangers à la réalite égyptienne et qui, en outre, cherchaient à rapprocher les deux cultures ${ }^{53}$. Diodore a dû connaître la récitation d'une litanie pour le mort (qu'il s'agisse de l'Apis ou d'un défunt humain), il a même pu assister à une dramatisation du voyage de l'âme vers l'au-delà. Il a confondu la représentation mimée d'un sujet imaginaire avec la réalité d'une pratique funéraire, car le jugement du mort par ses voisins, tel que le transmet Diodore, est absolument improbable ${ }^{54}$. Il faut en outre signaler qu'il devait s'agir en grande partie de cérémonies dont l'accès était réservé aux seuls prêtres, ce qui implique que les connaissances de Diodore seraient indirectes.

La seconde provient de la propre idéologie de l'auteur déterminée par de nombreuses influences dont celle d'Évhémère, qui semble importante dans notre cas ${ }^{55}$. L'Égypte pour Diodore est le pays dont la culture est la plus ancienne ${ }^{56}$, la patrie d'Osiris-Dionysos, être humain divi-

53 D'autres exemples dans BURTON, p. 13 sq.

54 Un jugement de ce genre semble très improbable en tenant compte des fortes dépenses pour le bien-être de la dépouille matérielle (construction du tombeau, momification, etc.) et des soigneuses précautions pour assurer le succès de l'âme dans la survie d'outre-tombe (textes magiques, rituels d'offrandes journaliers, etc.). Des dispositions si minutieusement conçues préalablement ne semblent pas pouvoir coexister avec la possibilité d'un résultat aléatoire dans un verdict de dernière heure.

55 En fait notre connaissance d'Évhémère doit beaucoup aux soins de Diodore qui transmet de longs fragments de son œuvre (dans V, 41-46 et VI, 1 - ap. Eus., $P E$, II, 2, 52-62 - repris comme fragments 1 et 2 de l'édition de G. VAllauri, Evemerus. Fragmenta et Testimonia, Turin, 1957, et disséminés d'après le sujet dans la récente édition de M. WINIARCzYK, Evhemerus Messenius Reliquiae, Stuttgart-Leipzig [Teubner] 1991). Mais l'influence d'Évhémère n'est pas la seule; homme de son temps, il subissait, par exemple, l'influence des visions de l'Égypte d'Hérodote (cf. note 25) ou de Platon (cf. récemment L. BRISSON, L'Égypte de Platon, in La Philosophie et l'Égypte, Les Etudes Philosophiques, 1987 (2/3), p. 153-168, ou J. McEvoy, Platon et la sagesse d'Égypte, in Kernos 6 [1993], p. 245-275). Diodore appartient donc à un courant d'écrivains grecs éblouis par le mirage égyptien, cf. C. FroIdEFond, Le mirage égyptien dans la littérature grecque d'Homère à Aristote, Aix-en-Provence, 1971, surtout p. 231 sq.; plus récemment F. HARTOG, Les Grecs égyptologues, in Annales(ESC), 41 (1986), p. 953 sq.

56 C'est une prémisse acceptée de façon générale depuis Hérodote. Voir un exemple excellent dans H.W. PARKE, Mighty Zeus, in Hermathena, 111 (1971), p. 24-33, qui montre comment l'antiquité des origines égyptiennes d'un culte 
nisé en raison de ses bienfaits civilisateurs ${ }^{57}$, une sorte de monarque hellénistique avant la lettre. Diodore, fils de son temps, s'attend à trouver en Égypte la réalité de ce qui, chez les autres peuples, est devenu fable, l'endroit où l'on se souvient de l'époque où les dieux étaient des hommes, espèce d'île Panchée visitable. Ces a priori l'amènent à interpréter comme réel ce qui, chez les Égyptiens, est tout aussi imaginaire que chez les Grecs : le jugement des morts 58 , le nocher (Charon), le Cerbère-Anubis et le dieu Hermès-Thot. Les cérémonies égyptiennes permettent, selon Diodore, d'expliquer les cérémonies grecques, plus récentes; de plus, elles donnent la clef pour voir matérialisé le modèle du pays de la véritable justice, utopie du bon gouvernement, Égypte dont une coutume salutaire permet même de priver de sépulture (le plus grand des châtiments) les injustes, grâce au verdict d'un tribunal terrestre qui semble infaillible ${ }^{59}$, et qui en cela est bien différent du tribunal aux erreurs constantes que nous dessine Platon dans le Gorgias (523b-e).

De nombreuses influences se superposent pour créer le miroir de Diodore, moins brillant que celui d'Hérodote ${ }^{60}$, mais tout aussi labyrinthique. Une dernière influence, non négligeable, provient de la propa-

(fussent-elles réelles ou supposées) servait comme argumentation lors des disputes entre sanctuaires oraculaires.

57 DIODORE, I, 17-18.

58 Le jugement des morts dans l'au-delà est un motif tardif chez les Grecs, étranger à la morale homérique et dans la ligne des croyances mystiques et philosophiques. HOMĖRE (Odyssée, XI, 568) présente Minos rendant justice dans l'Hadès, mais il agit en tant que roi (en fait il est le monarque défunt le plus puissant qui habite dans le royaume des morts) et rien ne permet de prouver que ses jugements se font selon un critère moral et déterminent l'entrée des défunts dans les enfers (cf. A. HEUBECK, A. HOEKSTRA, Commentary on Homer's Odyssey, II, Oxford, 1989, p. 111). Platon (Apol., 41a; Gorg., $523 \mathrm{sq}$.) est le véritable transmetteur de la croyance aux juges infernaux qui déterminent selon les actes réalisés au cours de la vie le destin futur du mort. L'emprunt égyptien semble en tout cas peu probable. En fait, le jugement des morts est un motif qui apparaît dans des cultures très diverses sur toute la planete, cf. S.G.F. BRANDON, The Judgement of the Dead : an Historical and Comparative Study of the Idea of a Post-mortem Judgement in the Major Religions, Londres, 1967.

59 Diodore , I, 92, 2 et 4-5. Les excellences de la justice égyptienne sont traitées aussi dans I, 75-76 et I, 48, 6.

60 Nous pensons évidemment à l'étude suggestive de F. HARTOG, Le miroir d'Herodote, Paris, 1980. 
gande politique des souverains du pays qu'il a visité. Son essai pour démontrer la prééminence de l'Égypte dans la direction des échanges culturels correspond bien à un courant d'idées à l'origine duquel semblent se trouver les rois lagides, qui voulaient donner la crédibilité du déjà vécu à leurs prétentions de domination politique et culturelle sur la Grèce ${ }^{61}$, et de prééminence dans le monde complexe des relations entre les royaumes hellénistiques.

En conclusion, il semble donc établi que les influences égyptiennes dans l'imaginaire grec de la mort tel que Diodore les rapporte semblent plus un emprunt supposé que réel ${ }^{62}$. Les emprunts idéologiques entre systèmes sociaux tellement différents et éloignés (même s'ils maintiennent des relations commerciales certaines) n'atteignent jamais une profondeur suffisante pour déterminer l'acceptation de fonctions et de noms tels que Diodore la transmet ${ }^{63}$; une acculturation de la catégo-

61 La syngeneia était une des bases de justification de la domination et Diodore ici ne fait que multiplier les exemples de parenté culturelle entre Égyptiens et Grecs, une façon de penser normale dans l'Égypte qu'il a visitée. Cf. récemment à ce sujet $\mathrm{P}$. VIDAL-NAQUET, Diodore et le vieillard de Crète, préface de Diodore de Sicile. Naissance des dieux et des hommes, Paris, 1991 (La Roue à Livres), p. XVIII.

62 La structure générale de l'idéologie funéraire égyptienne demeure profondément étrangère à la grecque malgré toutes les ressemblances ponctuelles (réelles ou imaginaires) que l'on peut constater (ce que font, nous l'avons vu, Diodore chez les Anciens, et Vermeule [citée en note 22], p. 71 sq., par exemple) parmi les chercheurs actuels). La sensibilité envers le corps mort est radicalement différente chez les deux peuples. Une adaptation écologique en Égypte a imposé le rituel de la momification (pour reproduire puis améliorer les résultats naturels de la conservation du cadavre dans le sable du désert), qui est devenu nécessaire dans l'idéologie de la survivance dans l'audelà. Pour les Grecs, au contraire, le corps du défunt n'accomplit pas une fonction idéologique qui requiert des soins spéciaux pour le conserver. Il faut se méfier des rapprochements ponctuels qui ne témoignent pas d'une véritable ressemblance dans la structure.

63 Il faut tout de même maintenir une position souple en ce qui concerne les emprunts et influences égyptiennes sur certains aspects culturels grecs. L'iconographie semble un véhicule de transmission de modèles égyptiens en Grèce qui ne peut pas être rejeté, surtout à des époques où la culture hellénique était très ouverte aux influences orientales. La transmission a de toute façon pu être indirecte dans les moments les plus reculés (tel semble le cas de l'emprunt, réadapté au chant XXI de l'Odyssée, de la preuve de l'arc dont le prototype revient aux démonstrations propagandistes de force physique du pharaon Aménophis II et de quelques-uns de ses successeurs, et qui put arriver en Grèce par la voie du Levant lors des diverses dominations 
rie de celle que Diodore illustre ne peut pas être causée par la seule influence de visiteurs en quête de connaissances, éblouis par le savoir des Égyptiens (même s'il s'agissait, comme le veut notre auteur, d'Orphée ou d'Homère, de bien improbables voyageurs). Il ne faut pas oublier, de surcroît, que l'Égypte avec une culture sacrée d'accès difficile, que ce soit pour les Égyptiens non lettrés ou pour les étrangers, ne disposait ni des outils ni de la volonté de la propager pour modifier la culture grecque, même à l'époque de la formation de celle-ci; il aurait fallu, par exemple, que les colonies égyptiennes situées en sol grec dont parle Diodore $(\mathrm{I}, 28,1-4)$ eussent été réelles ce qui semble à présent, malgré quelques auteurs ${ }^{64}$, aussi imaginaire que la barque de Charon.

Universidad de La Laguna

Departamento de Prehistoria, Antropología e Historia Antigua E - 38071 LA LAGUNA (TENERIFE) Islas Canarias

Francisco Diez de Velasco Miguel Angel Molinero Polo

égyptiennes, cf. W. BURKert, Von Amenophis II. Zur Bogenprobe des Odysseus, in Grazer Beiträge, 1 [1973], p. 69 sq.) et plus directe (artisans grecs voyageant en Égypte, artisans étrangers installés en territoire grec) à partir de l'époque de la koiné artistique orientalisante (cf. le paragraphe VI de KA. SHEEDy, A Prothesis Scene from the Analatos Painter, in MDAI(A), 105 (1990), p. 135 sq.; consulter aussi H. METZGER, Problèmes de langage iconographique grec, in CRAI (1992), p. 139 sq.; E. BRUNNER-TRAUT, Gelebte Mythen, Darmstadt, 1981; S.P. MoRRIs, Daidalos and the Origins of Greek Art, Princeton, 1990, ou W. BURKERT, The Orientalizing Revolution, Harvard Univ. Press, 1992, p. 9 sq.). Le sujet est ouvert mais il faut se méfier des excès.

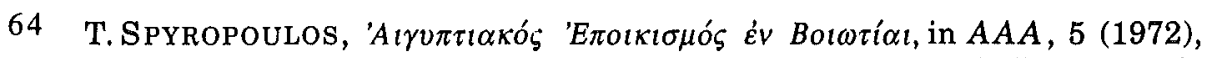
p. 16 sq.; B ERNAL, 1991 (cité en note 1), p. 78-153. Diodore a subi l'influence de la propagande lagide (cf. note 61). Ceci ne veut pas dire que les Grecs n'aient pas eu une connaissance de l'Égypte dès les époques les plus reculées, Cf. S. PERNIGOTTI, I più antichi rapporti tra l'Egitto e $i$ Greci, in Egitto e società antica. Atti Conv. Torino 1984, Milan, 1985, p. 77 sq.; J. VERCOUTTER, L'Égypte et le monde égéen préhellénique, Le Caire, 1956, ou P. LEVÊQUE, Colonisation grecque et syncrétisme, in Les syncrétismes dans les religions grecque et romaine, Coll. Strasbourg 1971, Paris, 1973, p. 56, mais il ne faut pas prendre très au sérieux les affirmations de parenté qu'exprime, par exemple, Platon (Ménéx., 245d). Le prestige de l'Égypte comme pays à la culture très ancienne provoquait la construction de prétendues filiations considérées comme logiques. 